Julius S. Bendat

J. S. Bendat Company 833 Moraga Drive Los Angeles, CA 90049

\title{
Spectral Techniques for Nonlinear System Analysis and Identification
}

\begin{abstract}
This article reviews some recent and current research work with emphasis on new recommended spectral techniques that can analyze and identify the optimum linear and nonlinear system properties in a large class of single-input/single-output nonlinear models by using experimentally measured input/output random data. This is done by showing how to replace these nonlinear models with equivalent multiple-input/ single-output linear models that are solvable by well-established practical procedures. The input random data can have probability density functions that are Gaussian or non-Gaussian with arbitrary spectral properties. Results in this article prove that serious errors can occur when conventional linear model analysis procedures are used to determine the physical properties of nonlinear systems. () 1993 John Wiley \& Sons, Inc.
\end{abstract}

\section{INTRODUCTION}

Nonlinear systems are defined as all systems that are not linear, namely, all systems where the output response data is not a linear function of the input excitation data. A large number of physical dynamic systems are naturally nonlinear or become nonlinear if the excitation to these systems is high enough. Thus, nonlinear systems are the usual situation rather than the exception in all branches of engineering and science. The complicated nature of nonlinear systems means that no universal approach is possible that can handle every type of nonlinear behavior.

During recent years, exciting advances have occurred in nonlinear system analysis and identification due to considering special types of nonlinear systems that can cover wide classes of importance, together with developing appropriate new techniques to solve the spectral decomposition problem for these nonlinear systems and the system identification problem for these nonlinear systems. Figure 1 shows ways to classify linear and nonlinear systems.

Nonlinear systems exhibit quite complicated and diverse behavior compared to linear systems. Much of the past research work in nonlinear system theory was directed toward defining a widely applicable mathematical model capable of converting a single-input data record into the many terms that comprise various possible nonlinear output records. The nonlinear formulation with the most universal application was found to be the Volterra series. The Volterra series assumes that the nonlinear response data can be expanded into a "power series with memory," with an $N$-integral kernel associated with each $N$ th order component. This approach leads to higher-order spectra such as bispectra and trispectra that are based on higher-order moments of the input data. 


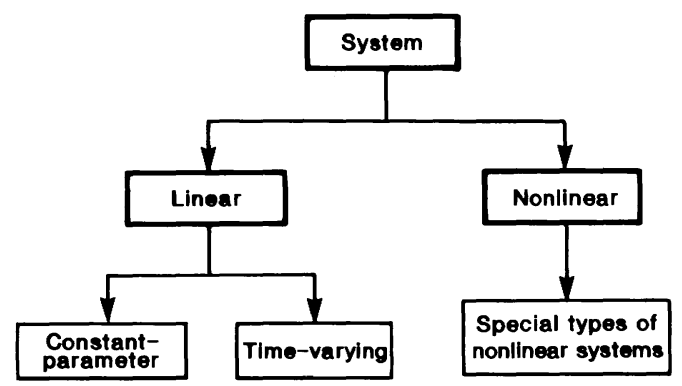

FIGURE 1 Classifications of linear and nonlinear systems.

Some of the major problems of Volterra series are:

1. sensitivity to a Gaussian assumption of the input data;

2. unreasonable computer processing requirements for kernels of order three or more;

3. difficulty in interpreting system behavior and properties of higher-order kernels;

4. large random errors in estimates that require excessive (often unobtainable) amounts of data for acceptable confidence limits.

These disadvantages motivated the research reported here.

In experimental work where input/output data are collected on physical systems, the main purpose is usually to validate a proposed physicalmathematical-engineering model of the linear and nonlinear system properties that might be present. This model can consist of component terms such as inertial, stiffness, and damping coefficients, together with excitation forces and restoring forces that represent the linear and/or nonlinear differential equations of motion of the system. The validation should seek to identify the nature of the physical process (qualitative properties) and the associated numerical parameters (quantitative properties) to determine from measured input/output data the properties of each term in a proposed nonlinear differential equation of motion.

Much past and current work in modal analysis arbitrarily assumes only linear differential equations of motion when nonlinear behavior is present, leading to results that give serious erroneous linear system properties as well as no knowledge of the actual nonlinear system properties. New practical techniques discussed in this paper are now available that can identify the correct linear system properties as well as the associated nonlinear system properties for single-degree-of-freedom (SDOF) nonlinear systems and multiple-degree-of-freedom (MDOF) nonlinear systems. In the future application of these new techniques will lead to better designed systems and more reliable predictions on their performance in severe environments.

The objectives of preliminary data analysis for a suspected nonlinear system are three-fold: detection that a nonlinearity is present or absent; identification of the type of nonlinearity; and estimation of some key system physical parameters.

A number of ways can be used to accomplish these objectives:

1. mathematical-physical knowledge of the nonlinear system operations;

2 . verification that the system violates the additive or homogeneous properties of linear systems;

3. calculation of input/output probability density functions (histograms);

4. calculation of input/output autospectral density functions;

5. calculation of ordinary coherence functions;

6. construction of restoring force/displacement relationships.

The first two ways are clear and require no discussion. For ideal linear systems subjected to a Gaussian random excitation, the response probability density function is theoretically Gaussian. However, if the system is nonlinear, the response will deviate from the Gaussian form. Hence, measurement of input/output probability density functions can provide a third simple way to detect nonlinear system operations that is also often able to help identify their nature as shown in Bendat [1990] and Bendat and Piersol [1986b]. For SDOF linear systems excited by broad-band random data, the autospectral density function of the response data is typically narrow-band in the vicinity of the resonant frequency. When nonlinearity is present, the response of the system may contain higher or lower harmonics relative to the resonant frequency band. Thus, measurement of input/output autospectra can provide a fourth way to detect and in some cases indicate the nature of the nonlinearity. Ordinary coherence functions can 
detect nonlinear behavior between a measured input and a measured output only when low values of coherence are not due to: extraneous noise in the input and/or output data; bias and/or random errors in the spectral estimates; or multiple inputs to the output besides the measured input. The patterns associated with restoring force/displacement relationships can help find and estimate important system physical parameters.

\section{LITERATURE REVIEW}

Some past nonlinear work of significance is listed below. This list is not intended to be exhaustive but consists only of certain books and papers that were helpful to the author.

1. Rice [1954] wrote an outstanding article that contains significant original work on zero-memory nonlinear systems, including a general approach to analyzing the response of nonlinear systems to random noise.

2. Deutsch's [1962] engineering book contains a broad treatment on nonlinear transformations of random processes devoted to applications in communication and control systems.

3. In the book that Haddad edited [1975] are found reprints of important articles analyzing zero-memory nonlinear systems and Volterra series with memory.

4. Broch's book [1975] contains reprints of technical articles written from 1961 to 1970 representing practical experimental studies for nonlinear systems subject to random vibrations.

5. A 1978 book by Marmarelis and Marmarelis, deals not only with the analysis of physiological systems using white-noise inputs but also with more general matters of nonlinear system analysis and identification.

6. The book by Schetzen [1980] is an outstanding treatise on the Volterra and Wiener theories of nonlinear systems that discusses these matters in great scientific detail.

7. Rugh [1981] wrote a book in which he develops many relations for input/output transformations through Volterra or Wiener series as represented by formulas in the time domain, the frequency domain, and from differential equations.

8. Rice and Fitzpatrick [1988, 1991] wrote two outstanding articles dealing with useful techniques for nonlinear system analysis and identification that were developed following the work by Bendat and Piersol [1982, 1986a], Bendat [1983], and Vugts and Bouquet [1985], but independently of other related work by Bendat [1985], Bendat and Palo [1989, 1990], and Bendat, Palo, and Coppolino [1990, 1992].

9. Contributions by Bendat to this field started in 1977 when he was asked by Jan Vugts of Shell Internationale Petroleum Maatschappij (SIPM) in Hague, The Netherlands, to conduct a mathematical study on how to apply spectral analysis techniques to nonlinear wave-force problems. New nonlinear wave-force models and special techniques were formulated that required verification by further computer simulation work and model basin experiments conducted for SIPM by Allan Piersol. Some results from this SIPM work are published in Bendat et al. [1982, 1983, 1986a] and Vugts and Bouquet [1985].

10. Extensions of this research have been conducted by Bendat from 1982 to date for the Naval Civil Engineering Laboratory (NCEL) in Port Hueneme, CA, with the active help of Paul Palo from the Ocean Structures Division of NCEL. Support for this work came from NCEL and from the Office of Naval Research (ONR). Computer assistance to verify important new ideas for nonlinear system identification was provided by Robert Coppolino of Measurement Analysis Corporation. New practical techniques have been developed for random data analysis of many types of nonlinear systems, together with new methods for identifying physical parameters in SDOF and MDOF nonlinear systems. Some results from this NCEL work are discussed in Bendat et al. [1985, 1989, 1990, 1992] and Bendat and Piersol [1993].

11. The most complete treatment in book form on these particular activities and other important nonlinear matters is by Bendat [1990]. This book contains new simple one-dimensional formulas that can replace complicated Volterra/Wiener 
multidimensional formulas. Relations are derived that can predict the separate linear and nonlinear output spectra when stationary random data or transient random data pass through single-input/singleoutput (SI/SO) nonlinear models with parallel linear, bilinear, and trilinear systems. New practical procedures are developed that show how to identify the nonlinear system amplitude and frequency properties in desired SI/SO nonlinear models by using equivalent multiple-input/singleoutput (MI/SO) linear models.

Engineering applications in Bendat [1990] include: response prediction of zero-memory and finite-memory nonlinear systems due to stationary random input data; the importance of modeling zero-memory nonlinear systems by optimum third-order polynomial least-squares approximations; analysis and identification of linear and nonlinear wave forces on slender structural members when the nonlinear wave forces involve squaring operations with sign on the wave velocity input; analysis and identification of linear and nonlinear drift forces on ships when the nonlinear drift forces are proportional to the squared wave envelope of the wave elevation input; practical ways to identify from measured input/output random data the physical parameters of mass, stiffness, and damping coefficients in proposed nonlinear differential equations of motion.

\section{NEW RESULTS}

Techniques discussed in this paper for solving nonlinear models offer many advantages compared to other nonlinear system analysis and identification techniques such as: (1) the Volterra and related Wiener nonlinear system theories; (2) the computation and applications of higher-order bispectra and trispectra; or (3) the use of autoregressive (AR), moving average (MA), or combined (ARMA) models. None of these alternative techniques provide desired linear and nonlinear system parameters appropriate for structuraltype problems as can be done by the new recommended methods. Some features of these new methods are:

1. The identification of the linear and nonlinear parts of nonlinear models can be imple- mented using established MI/SO linear procedures and computer programs.

2. The solution gives nonlinear system amplitude properties separate from nonlinear system frequency properties.

3. Relative spectral response properties from different nonlinear terms can be quantified by using appropriate coherence functions.

4. Results are independent of the input or output probability distributions or spectral properties.

5. Results are independent of the input excitation levels as required for valid nonlinear models.

6. Results are relatively insensitive to extraneous noises at input and output points if noise-to-signal power ratios are less than $10 \%$.

\section{TYPES OF NONLINEAR SYSTEMS}

Special types of nonlinear systems of wide interest are:

1. zero-memory and finite-memory nonlinear systems;

2. bilinear and trilinear systems in a Volterra series;

3. square-law and cubic nonlinear systems;

4. parallel linear and nonlinear systems.

A zero-memory nonlinear system, shown in Fig. 2, is a system where the output $y(t)$ at any time $t$ is a nonlinear function $g(x)$ of the input $x(t)$ at the same instant of time, namely,

$$
y(t)=g[x(t)] .
$$

The function $g(x)$ is a function of $x$; it is not a function of $t$. No weighting is done of past inputs as with linear systems defined by a unit impulse response function $h(\tau)$ in the time domain, or by its associated frequency response function $H(f)$ in the frequency domain.

Nonlinear system means that for any constants $a_{1}, a_{2}$, and any inputs $x_{1}, x_{2}$, one obtains the inequality

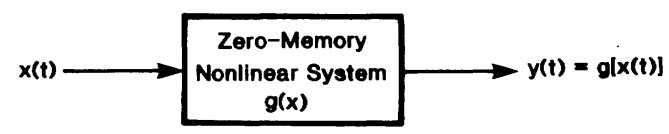

FIGURE 2 Zero-memory nonlinear system. 


$$
g\left(a_{1} x_{1}+a_{2} x_{2}\right) \neq a_{1} g\left(x_{1}\right)+a_{2} g\left(x_{2}\right) .
$$

This equation violates both the additive and homogeneous properties of linear systems. The system is a constant-parameter nonlinear system if the response of the system is independent of the particular time of use, namely, if the input $x(t)$ becomes $x(t+\tau)$, then the output $y(t)$ becomes $y(t+\tau)$. If the system is a constant-parameter nonlinear system and if the input $x(t)$ represents a stationary random process, then the output $y(t)$ will also represent a stationary random process. For these cases, $y(t)=g[x(t)]$ will give $y(t+\tau)=g[x(t+\tau)]$ with the output autocorrelation function $R_{y y}(\tau)$ and the input/output cross-correlation function $R_{x y}(\tau)$ defined by

$$
R_{y y}(\tau)=E[y(t) y(t+\tau)]=E\{g[x(t)] g[x(t+\tau)]\}
$$

$R_{x y}(\tau)=E[x(t) y(t+\tau)]=E\{x(t) g[x(t+\tau)]\}$.

Examples of zero-memory nonlinear systems include systems such as the following that are formulated and illustrated in Bendat [1990]: two-slope system; clipped system; hard-clipped system; dead-zone system; square-law system; cubic system; square-law system with sign; hardening spring system and softening spring system.

Bendat [1990] discusses three previously known theorems for predicting desired input/output relations when stationary random data pass through specified zero-memory nonlinear systems. Theorem 1 applies to arbitrary input data and predicts the output probability density function from knowledge of the input probability density function. Theorem 2 applies only to Gaussian input data and predicts the output autocorrelation function from knowledge of the input autocorrelation function. Theorem 3 applies also only to Gaussian input data and predicts the input/output cross-correlation function from the input autocorrelation function.

When finite-memory operations are desired along with zero-memory nonlinear systems, they can often be modeled by inserting a constantparameter linear system either "before" and/or "after" the zero-memory nonlinear system. Cases of a linear system that is after the zeromemory nonlinear system represent cases of physical interest that can be solved for nonGaussian input data and are the only cases considered in this paper. Bendat [1990] discusses

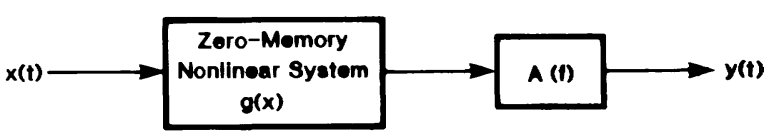

FIGURE 3 Finite-memory nonlinear system.

other cases of a linear system before the zeromemory nonlinear system. Figure 3 shows a finite-memory nonlinear system where a linear system with frequency response function $A(f)$ is after the zero-memory nonlinear system defined by $g(x)$.

A general third-order nonlinear system input/ output model with parallel linear, bilinear, and trilinear systems is shown in Fig. 4. These bilinear and trilinear systems represent the second and third terms in a Volterra series. Higher-order terms are strongly discouraged because of their complexity.

Linear systems are defined by a first-order frequency response function that is a single Fourier transform of a first-order weighting function. Bilinear systems are defined by a second-order frequency response function that is a double Fourier transform of a second-order weighting function. Trilinear systems are defined by a thirdorder frequency response function that is a triple Fourier transform of a third-order weighting function. The multidimensional bilinear and trilinear functions are difficult to compute and hard to interpret so that applications of these procedures should be conducted only when the alternative techniques discussed in this paper that require only one-dimensional functions are not deemed suitable. Derivations of the required multidimensional formulas to obtain optimum bilinear and trilinear systems from measured data are contained in Bendat [1990].

Square-law and cubic nonlinear systems are special cases of bilinear and trilinear systems that can approximate many finite-memory nonlinear

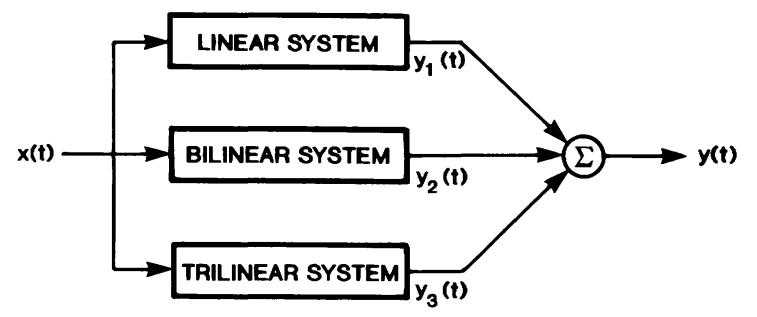

FIGURE 4 Third-order nonlinear system input/output model. 


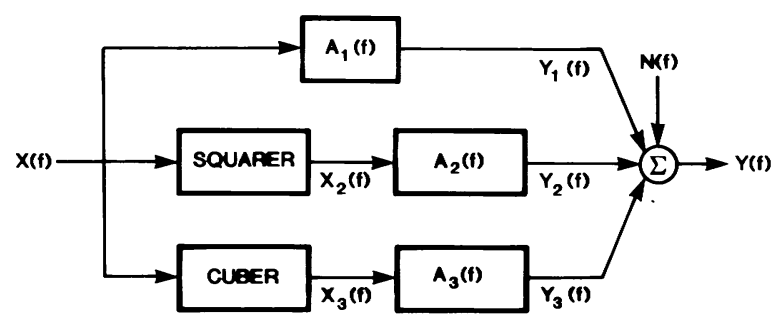

FIGURE 5 Case 1 nonlinear model.

systems and can be analyzed using only onedimensional functions. The approximation is valid whenever the zero-memory nonlinear system can be represented by an optimum leastsquares third-order polynomial.

Figure 5 is called a Case 1 nonlinear model [Bendat, 1990] where the $A(f)$ linear operations are $A$ for after the squarer and cuber operations. In Fig. $5, X(f)$ is the Fourier transform of the input $x(t), X_{2}(f)$ is the Fourier transform of the squarer output $x^{2}(t)$, and $X_{3}(f)$ is the Fourier transform of the cuber output $x^{3}(t)$. The record $X(f)$ can be Gaussian or non-Gaussian; however, the records $X_{2}(f)$ and $X_{3}(f)$ will always be nonGaussian. The Case 1 nonlinear model is the model that should be used whenever appropriate because it is more general and easier to solve than Case 2 nonlinear models where linear systems precede the squarer and cuber operations. Extended Case 1 type nonlinear models occur as shown later can also be solved when the squarer is replaced by an arbitrary zero-memory nonlinear system, and the cuber is replaced by another arbitrary zero-memory nonlinear system, provided that Fourier transforms $X_{2}(f)$ and $X_{3}(f)$ are known for the outputs of these two nonlinear systems.

The key to solving Case 1 type nonlinear models for the three $A(f)$ frequency response functions from measured input/output data is to

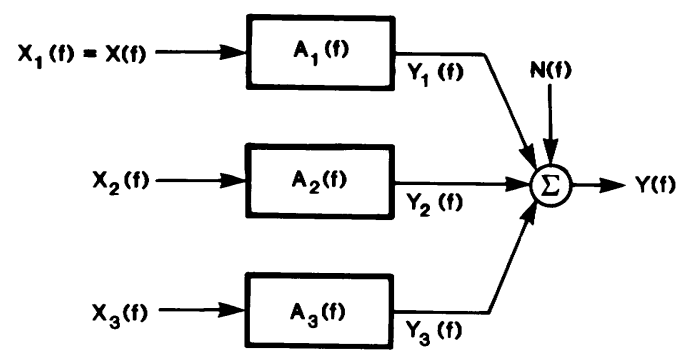

FIGURE 6 Three-input/single-output linear model equivalent to Fig. 5 where the inputs can be correlated.

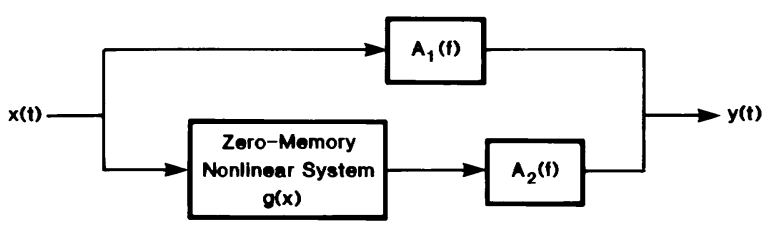

FIGURE 7 Single-input/single-output nonlinear model of a linear system in parallel with one nonlinear system.

recognize that the SI/SO nonlinear model of Fig. 5 can be treated as a three-input/single-output linear model shown in Fig. 6, with the three (correlated) records $X_{1}(f), X_{2}(f)$, and $X_{3}(f)$ computed from the original measured input data. Figure 6 can then be solved for the three frequency response functions $A_{1}(f), A_{2}(f)$, and $A_{3}(f)$ by using standard $\mathrm{MI} / \mathrm{SO}$ linear techniques [Bendat and Piersol, 1986b, 1993] where input data $X(f)$ can be Gaussian or non-Gaussian.

\section{NEW METHODS TO SOLVE PARALLEL LINEAR AND NONLINEAR SYSTEMS}

A general methodology is developed in Bendat [1990] for analyzing arbitrary linear systems in parallel with arbitrary nonlinear systems. An important class of such problems that is applicable to many physical situations is the SI/SO nonlinear model of Fig. 3 when the input $x(t)$, which may be Gaussian or non-Gaussian, passes through parallel linear and finite-memory nonlinear systems as shown in Fig. 7. The frequency response functions $A_{1}(f)$ and $A_{2}(f)$ represent arbitrary constant-parameter linear systems, and $g(x)$ is any specified zero-memory nonlinear system.

A more general SI/SO nonlinear model than Fig. 7 can be constructed that involves one linear system $A_{1}(f)$ in parallel with two different finitememory nonlinear systems, namely, $g_{2}(x)$ followed by $A_{2}(f)$ and $g_{3}(x)$ followed by $A_{3}(f)$ (Fig. $8)$. Figure 7 is a special case when $g_{3}(x)$ is zero. Also, the Case 1 nonlinear model of Fig. 5 is a special case when $g_{2}(x)=x^{2}$ and $g_{3}(x)=x^{3}$. Figure 8 is clearly able to cover a much larger class of physical problems and applications than either Figs. 5 or 7.

Two engineering problems of great importance for many applications [Bendat, 1990] involve the nonlinear model in Fig. 8, namely, (1) the spectral decomposition problem and (2) the 


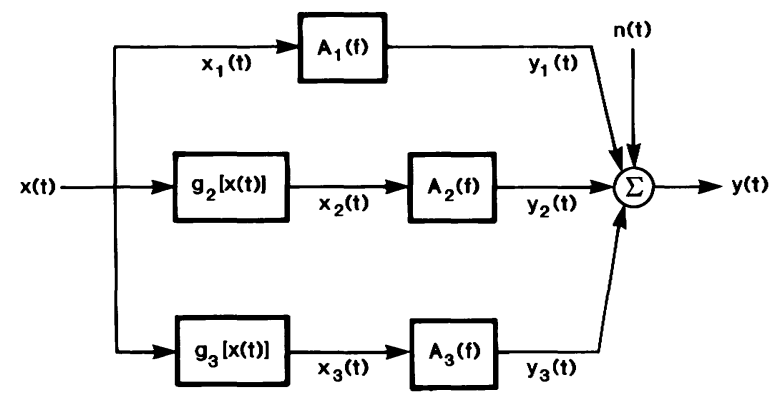

FIGURE 8 Single-input/single-output nonlinear model of a linear system in parallel with two nonlinear systems.

system identification problem. The spectral decomposition problem is straightforward to solve. The more difficult problem is the system identification problem.

\section{Spectral Decomposition Problem}

Given the frequency functions $A_{1}(f), A_{2}(f)$, $A_{3}(f)$, and given also the zero-memory nonlinear functions $g_{2}(x)$ and $g_{3}(x)$, from measurement only of the input $x(t)$, determine the spectral properties of the three outputs $y_{1}(t), y_{2}(t)$, and $y_{3}(t)$ shown in Fig. 8. If the total output $y(t)$ is measured as well as $x(t)$, determine also the spectral properties of the extraneous noise $n(t)$.

\section{System Identification Problem}

From simultaneous measurements of both the input $x(t)$ and the total output $y(t)$, identify the optimum frequency response functions $A_{1}(f), A_{2}(f)$, and $A_{3}(f)$ in Fig. 8 to minimize the autospectrum of $n(t)$. Show that the resulting noise $n(t)$ will be automatically uncorrelated with $x(t), y_{1}(t), y_{2}(t)$, and $y_{3}(t)$ when the optimum $A(f)$ systems are computed.

Some formulas and procedures will now be stated for solving the system identification problem in the general nonlinear model of Fig. 8 when the input data are non-Gaussian. Gaussian input cases are special cases of these formulas.

Consider the general SI/SO nonlinear model of Fig. 8 with three parallel paths where the input data $x(t)$ can be non-Gaussian. Let $g_{2}[x(t)]$ and $g_{3}[x(t)]$ be two known or proposed zero-memory nonlinear transformations of $x(t)$. Let

$$
x_{1}(t)=x(t), \quad x_{2}(t)=g_{2}[x(t)], \quad x_{3}(t)=g_{3}[x(t)]
$$

represent the three (usually correlated) input records to the three linear systems $A_{1}(f), A_{2}(f)$, and $A_{3}(f)$, respectively. The three associated (usually correlated) output records from these systems are denoted by $y_{1}(t), y_{2}(t)$, and $y_{3}(t)$, respectively. To complete the model, the term $n(t)$ represents extraneous uncorrelated output noise and $y(t)$ represents the total output data from the system.

Figure 8 (like Fig. 5 with respect to Fig. 6) can be replaced by its equivalent three-input/singleoutput linear model shown in Fig. 9 where the capital letter quantities are Fourier transforms of associated small letter quantities. To be specific, in Fig. 9, $X_{1}(f), X_{2}(f)$, and $X_{3}(f)$ are Fourier transforms of $x_{1}(t), x_{2}(t)$, and $x_{3}(t)$, respectively; $Y_{1}(f), Y_{2}(f)$, and $Y_{3}(f)$ are Fourier transforms of $y_{1}(t), y_{2}(t)$, and $y_{3}(t)$, respectively; $N(f)$ is the Fourier transform of $n(t)$ and $Y(f)$ is the Fourier transform of $y(t)$. The same systems $A_{1}(f)$, $A_{2}(f)$, and $A_{3}(f)$ are in both Figs. 8 and 9. Recognition of this equivalence is a significant achievement for SI/SO nonlinear models like Fig. 8 because the $A(f)$ systems in Fig. 9 (same as Fig. 6) can be identified by straightforward MI/SO linear techniques. These procedures [Bendat and Piersol, 1986b; Bendat and Palo, 1990] are applicable for input data that can be Gaussian or non-Gaussian, and can be extended to apply to any arbitrary number of parallel nonlinear paths in Fig. 8 with different $g[x(t)]$ and $A(f)$ in each nonlinear path.

The basis of the MI/SO procedures for identifying the system properties in Fig. 9 is to change the (generally correlated) input records using conditioned spectral density techniques so that the first input $U_{1}(f)=X_{1}(f)$ is left alone, the second input $X_{2}(f)$ becomes $U_{2}(f)=X_{2 \cdot 1}(f)$ where the linear effects of $X_{1}(f)$ are removed from $X_{2}(f)$, and the third input $X_{3}(f)$ is changed to $U_{3}(f)=X_{3 \cdot 2 !}(f)$ where the linear effects of

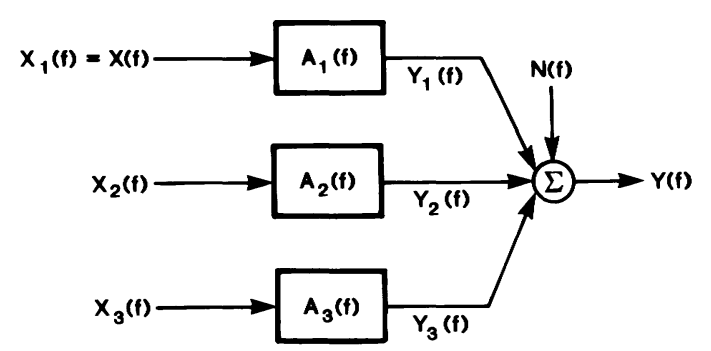

FIGURE 9 Three-input/single-output linear model equivalent to Fig. 8 where the inputs can be correlated. 


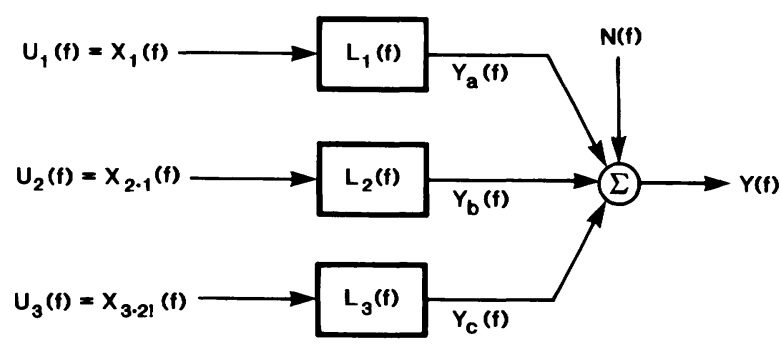

FIGURE 10 Revised three-input/single-output linear model equivalent to Fig. 9 where the inputs are mutually uncorrelated.

both $X_{1}(f)$ and $X_{2}(f)$ are removed from $X_{3}(f)$. These conditioned input records will then be mutually uncorrelated and become the inputs to the revised three-inputs/single-output linear model in Fig. 10. The noise output record $N(f)$ and the total output record $Y(f)$ are the same as before. However, the three previous (generally correlated) output records $Y_{1}(f), Y_{2}(f)$, and $Y_{3}(f)$ are now replaced by three new mutually uncorrelated output records $Y_{a}(f), Y_{b}(f)$, and $Y_{c}(f)$, and the three previous linear systems $A_{1}(f), A_{2}(f)$, and $A_{3}(f)$ are now replaced by three new linear systems denoted by $L_{1}(f), L_{2}(f)$, and $L_{3}(f)$. Identification of $L(f)$ systems by $S I / S O$ linear techniques then gives the $A(f)$ systems by algebraic equations. Appropriate ordinary coherence functions can be computed easily that state at each frequency the percentage of the total output autospectral density function due to each uncorrelated input record in Fig. 10.

The optimum overall linear system frequency response function $H_{o}(f)$ associated with Fig. 8 (considered solely as SI/SO linear model with output noise) is given by the formula

$$
H_{o}(f)=\left[G_{x y}(f) / G_{x x}(f)\right]=L_{1}(f)
$$

where $G_{x x}(f)$ is the autospectral density function of the input record $x(t)$ and $G_{x y}(f)$ is the crossspectral density function between the input record $x(t)$ and the total output record $y(t)$. This optimum linear system $H_{o}(f)$ is the same as the linear system $L_{1}(f)$ in Fig. 10. However, in general, as shown in Eq. 13, the linear system $L_{1}(f)$ in Fig. 10 is not the same as the linear system $A_{1}(f)$ in Figs. 8 and 9, namely,

$$
L_{1}(f) \neq A_{1}(f) .
$$

Thus, computation of $H_{o}(f)$ by conventional linear modal analysis techniques can give errone- ous estimates of the desired linear frequency response function $A_{1}(f)$ in SI/SO nonlinear models like Fig. 8, as well as no information on the other possible linear systems $A_{2}(f)$ and $A_{3}(f)$ that follow the specified zero-memory nonlinear operations in Fig. 8. This is a serious limitation in current modal analysis work that can be corrected by employing these new techniques.

General formulas for these $L$-systems and $A$ systems are as follows:

$$
L_{1}(f)=\left[G_{1 y}(f) / G_{11}(f)\right]=\left[G_{u_{1} y}(f) / G_{u_{1} u_{1}}(f)\right]
$$

$L_{2}(f)=\left[G_{2 y \cdot 1}(f) / G_{22 \cdot 1}(f)\right]=\left[G_{u_{2} y}(f) / G_{u_{2} u_{2}}(f)\right]$

$$
L_{3}(f)=\left[G_{3 y \cdot 2 !}(f) / G_{33 \cdot 2 !}(f)\right]=\left[G_{u_{3} y}(f) / G_{u_{3} u_{3}}(f)\right]
$$

$$
A_{3}(f)=L_{3}(f)
$$

$$
\begin{aligned}
A_{2}(f)= & L_{2}(f)-\left[G_{23 \cdot 1}(f) / G_{22 \cdot 1}(f)\right] A_{3}(f) \\
A_{1}(f)= & L_{1}(f)-\left[G_{12}(f) / G_{11}(f)\right] A_{2}(f) \\
& -\left[G_{13}(f) / G_{11}(f)\right] A_{3}(f) .
\end{aligned}
$$

In the analysis of MI/SO linear systems, various coherence functions should always be computed to quantify the goodness-of-fit of the chosen model and the numerical results. This is an important step because it provides an absolute measure on how well each term of the model fits the data at specific frequencies of interest. This type of information is not available in time-domain system identification techniques.

Care must be taken when calculating and interpreting coherence functions for correlated inputs. In these cases, the recommended practice is to uncorrelate the inputs by conditioning, then to compute ordinary coherence functions that can be inspected individually and summed algebraically to obtain the goodness-of-fit for the entire model. This was the reason for changing the problem from Fig. 9 into Fig. 10 with the uncorrelated inputs $U_{1}(f), U_{2}(f)$, and $U_{3}(f)$, and associated uncorrelated outputs $Y_{a}(f), Y_{b}(f)$, and $Y_{c}(f)$.

The component and total output spectral density functions in Fig. 10 are given by the formulas

$$
G_{y_{a} y_{a}}(f)=\left|L_{1}(f)\right|^{2} G_{u_{1} u_{1}}(f)=\gamma_{u_{1} y}^{2}(f) G_{y y}(f)
$$

$G_{y_{b} y_{b}}(f)=\left|L_{2}(f)\right|^{2} G_{u_{2} u_{2}}(f)=\gamma_{u_{2} y}^{2}(f) G_{y y}(f)$ 


$$
\begin{aligned}
G_{y_{c} y_{c}}(f)= & \left|L_{3}(f)\right|^{2} G_{u_{3} u_{3}}(f)=\gamma_{u_{3} y}^{2}(f) G_{y y}(f) \\
G_{y y}(f)= & G_{y_{a} y_{y}}(f)+G_{y_{b} y_{b}}(f)+G_{y_{c} y_{c}}(f) \\
& +G_{n n}(f) .
\end{aligned}
$$

The first ordinary coherence function $\gamma_{u_{1} y}^{2}(f)$ in Fig. 10 is the ordinary coherence function between the input $U_{1}(f)=X_{1}(f)=X(f)$ and the total output $Y(f)$. It states the proportion of the output spectrum $G_{y y}(f)$ that is due to $U_{1}(f)$ passing through the linear system $L_{1}(f)$ that represents all the possible ways $U_{1}(f)$ can get to $Y(f)$, where

$$
\gamma_{u_{1} y}^{2}(f)=\left[\left|G_{u_{1} y}(f)\right|^{2} / G_{u_{1} u_{1}}(f) G_{y y}(f)\right] .
$$

The second ordinary coherence function $\gamma_{u_{2} y}^{2}(f)$ in Fig. 10 is the ordinary coherence function between the input $U_{2}(f)=X_{2 \cdot 1}(f)$ and the total output $Y(f)$. It states the proportion of the output spectrum $G_{y y}(f)$ that is due to $U_{2}(f)$ passing through the linear system $L_{2}(f)$ that represents all of the possible ways $U_{2}(f)$ can get to $Y(f)$, where

$$
\gamma_{u_{2} y}^{2}(f)=\left[\left|G_{u_{2} y}(f)\right|^{2} / G_{u_{2} u_{2}}(f) G_{y y}(f)\right] .
$$

The third ordinary coherence function $\gamma_{u_{3} y}^{2}(f)$ in Fig. 10 is the ordinary coherence function between the input $U_{3}(f)=X_{3 \cdot 2 !}(f)$ and the total output $Y(f)$. It states the proportion of the output spectrum $G_{y y}(f)$ that is due to $U_{3}(f)$ passing through the linear system $L_{3}(f)$ that represents all of the possible ways $U_{3}(f)$ can get to $Y(f)$, where

$$
\gamma_{u_{3} y}^{2}(f)=\left[\left|G_{u_{3} y}(f)\right|^{2} / G_{u_{3} u_{3}}(f) G_{y y}(f)\right] .
$$

The multiple coherence function in Fig. 10 is now given by

$$
\gamma_{y: x}^{2}(f)=\gamma_{u_{1} y}^{2}(f)+\gamma_{u_{2} y}^{2}(f)+\gamma_{u_{3} y}^{2}(f) .
$$

This multiple coherence function is the algebraic sum of the three component ordinary coherence function terms and states the goodness-of-fit of the postulated model at each frequency. This formula does not involve the computation of any partial coherence functions. Good models occur at desired frequencies when the multiple coherence function is close to unity.

The associated uncorrelated output noise spectrum $G_{n n}(f)$ that represents all possible devi- ations from the postulated model in Fig. 10 is given by the formula

$$
G_{n n}(f)=\left[1-\gamma_{y: x}^{2}(f)\right] G_{y y}(f) .
$$

Good models occur at desired frequencies when $G_{n n}(f)$ is close to zero.

A cumulative coherence function is defined in Fig. 10 at each frequency by partial sums of the component ordinary coherence functions. The first, second, and third cumulative coherence functions are defined by:

first cumulative coherence function $=\gamma_{u_{1} y}^{2}(f)$

second cumulative coherence function

$$
=\gamma_{u_{1} y}^{2}(f)+\gamma_{u_{2} y}^{2}(f)
$$

third cumulative coherence function

$$
=\gamma_{u_{1} y}^{2}(f)+\gamma_{u_{2} y}^{2}(f)+\gamma_{u_{3} y}^{2}(f) .
$$

The first cumulative coherence function identifies the proportion of the output spectrum $G_{y y}(f)$ due to $U_{1}(f)$ passing through the linear system $L_{1}(f)$. The second cumulative coherence function identifies the proportion of the output spectrum $G_{y y}(f)$ due to $U_{1}(f)$ and $U_{2}(f)$ passing through $L_{1}(f)$ and $L_{2}(f)$. The third cumulative coherence function identifies the proportion of the output spectrum $G_{y y}(f)$ due to $U_{1}(f), U_{2}(f)$, and $U_{3}(f)$ passing through $L_{1}(f), L_{2}(f)$, and $L_{3}(f)$. This third cumulative coherence function is the same as the multiple coherence function in Eq. 21. Inspection of the cumulative coherence functions allows for quantitative evaluation of the model at each frequency for various combinations of terms.

\section{SUMMARY OF NEW PROCEDURES}

In summary, the following steps should be carried out to determine the linear and nonlinear system physical properties in SI/SO nonlinear models such as Figs. 5 or 8 .

\section{Step 1}

Perform a preliminary analysis of the measured data to detect the most probable nonlinearities that can be described by selected zero-memory nonlinear operations such as $g_{2}(x)$ and $g_{3}(x)$ (Fig. $8)$, or by other nonlinear terms that may be indi- 
cated by consideration of possible nonlinear differential equations of motion. Propose the nonlinear model of Fig. 8, or variations thereof, where the unknown $A$-systems are to be determined from measured non-Gaussian input data and measured total output data. When the nonlinear operations are unknown and/or difficult to identify, with input data that can be non-Gaussian, propose the model of Fig. 5 with a squarer and cuber as third-order approximations to the unknown $g_{2}(x)$ and $g_{3}(x)$. Include as many nonlinear terms with their respective $A(f)$ frequency response functions in the proposed SI/SO nonlinear model and later equivalent reverse dynamic $\mathrm{MI} / \mathrm{SO}$ linear model as appears reasonable for the following reasons:

1. This technique will correctly determine that there are no $A(f)$ frequency response function for nonlinear terms not present in the physical model, thus indicating that the selected mathematical model can properly represent the physical situation.

2. The inclusion of additional nonlinear terms does not bias the other results, thus indicating that results do not change with different models.

3. The only cost for obtaining this extra information is slightly increased computer time to analyze the additional nonlinear terms.

\section{Step 2}

Convert the proposed SI/SO nonlinear model into an equivalent MI/SO linear model. As discussed by Bendat [1990], when dealing with a large class of nonlinear differential equations of motion, the systems can be identified by interchanging the roles of the measured input and output data to create a reverse dynamic MI/SO linear model. This significant idea changes the class of SI/SO nonlinear models with feedback into equivalent MI/SO linear models without feedback.

\section{Step 3}

Precompute a time series for each proposed zero-memory nonlinear input term such as $g_{2}[x(t)]$ and $g_{3}[x(t)]$. Compute Fourier transforms of each of these terms.

\section{Step 4}

For general non-Gaussian input data, compute all of the needed original and conditioned spectral density functions to solve for the uncorrelated input data in the SI/SO nonlinear model of Fig. 10. Compute similar results for alternate models with different nonlinearities.

\section{Step 5}

Assuming here that there are only two nonlinear paths, compute the three frequency response functions $L_{i}(f), i=1,2,3$, for the uncorrelated inputs in Fig. 10; then algebraically solve for the three frequency response functions $A_{i}(f), i=1$, 2, 3, for the correlated inputs in Fig. 9. General mathematical formulas for non-Gaussian input data are listed in Eqs. 8-13.

\section{Step 6}

Compute the component and total output spectral density functions by relations such as Eqs. 14-17 and interpret the results. Compute and interpret similar results for alternate models with different nonlinearities.

\section{Step 7}

Compute the ordinary coherence functions, the multiple coherence function and the output noise spectrum by relations such as Eqs. 18-22 and interpret the results. Compute and interpret similar results for alternate models.

\section{Step 8}

Compute the cumulative coherence functions by relations such as Eqs. 23-25 and interpret the results. Compute and interpret similar results for alternate models.

\section{Step 9}

Conduct standard statistical error analysis evaluations on the various computed estimates using formulas by Bendat [1990] and Bendat and Piersol [1986b, 1993].

Engineering applications for nonlinear wave force problems and nonlinear drift force problems of the just reviewed new MI/SO techniques 
for Gaussian and non-Gaussian input data are discussed by Bendat [1990], Bendat et al. [1985, 1986a, 1989] and Vugts and Bouquet [1985]. These applications are representative of many other problems that can occur in different physical fields where SI/SO nonlinear models of parallel linear and nonlinear systems can be replaced by equivalent MI/SO linear models. Advanced computer simulation studies have been conducted for NCEL based upon reverse dynamic system MI/SO ideas, that yield new ways to determine linear and nonlinear system physical parameters in SDOF and MDOF nonlinear differential equations of motion from measured input/output data [Bendat et al., 1990, 1992]. Some of these important practical new results from research work for NCEL are illustrated by examples in the last chapter of Bendat and Piersol [1993].

This article contains results from research work sponsored by NCEL and the ONR for development of new methods for random data analysis and identification of nonlinear systems by the J. S. Bendat Company. The author thanks Paul A. Palo of the Ocean Structures Division at NCEL for his constructive assistance in advancing this work and in reviewing this article.

\section{REFERENCES}

Bendat, J. S., 1983, "Statistical Errors for Nonlinear System Measurements Involving Square-Law Operations," Journal of Sound and Vibration, Vol. 90, pp. 275-282.

Bendat, J. S., March 1985, "Nonlinear System Dynamic Analysis Using Random Data," CR 85.006, Naval Civil Engineering Laboratory, Port Hueneme, CA.

Bendat, J. S., 1990, Nonlinear System Analysis and Identification from Random Data, Wiley-Interscience, New York.

Bendat, J. S., and Palo, P. A., September 1989, “Nonlinear System Stochastic Techniques for Ocean Engineering Applications," CR 89.018, Naval Civil Engineering Laboratory, Port Hueneme, CA.

Bendat, J. S., and Palo, P. A., 1990, "Practical Techniques for Nonlinear System Analysis and Identification," Sound and Vibration, Vol. 24, pp. 28-34.

Bendat, J. S., Palo, P. A., and Coppolino, R. N., March 1990, "Identification Techniques for Nonlin- ear Differential Equations of Motion," TN-1810, Naval Civil Engineering Laboratory, Port Hueneme, CA.

Bendat, J. S., Palo, P. A., and Coppolino, R. N., 1992, "A General Identification Technique for Nonlinear Differential Equations of Motion," Probabilistic Engineering Mechanics, Vol. 7, pp. 43-61.

Bendat, J. S., and Piersol, A. G., 1982, "Spectral Analysis of Nonlinear Systems Involving SquareLaw Operations,' Journal of Sound and Vibration, Vol. 81, pp. 199-213.

Bendat, J. S., and Piersol, A. G., 1986a, "Decomposition of Wave Forces into Linear and Nonlinear Components," Journal of Sound and Vibration, Vol. 106, pp. 391-408.

Bendat, J. S., and Piersol, A. G., 1986b, Random Data: Analysis and Measurement Procedures, 2nd ed., Wiley-Interscience, New York.

Bendat, J. S., and Piersol, A. G., 1993, Engineering Applications of Correlation and Spectral Analysis, 2nd ed. Wiley-Interscience, New York.

Broch, J. T., 1975, Nonlinear Systems and Random Vibration, Bruel \& Kjaer, Naerum, Denmark.

Deutsch, R., 1962, Nonlinear Transformations of Random Processes, Prentice-Hall, Englewood Cliffs, NJ.

Haddad, A. H., Editor, Nonlinear Systems, pp. 8-22, 59-63, 167-186, 1975, Dowden, Hutchinson \& Ross, Stroudsburg, PA.

Marmarelis, P. Z., and Marmarelis, V. Z., 1978, Analysis of Physiological Systems: The White-Noise Approach, Plenum Press, New York.

Rice, S. O., "Mathematical Analysis of Random Noise," in N. Wax, Selected Papers on Noise and Stochastic Processes, pp. 133-294, 1954, Dover, New York.

Rice, H. J., and Fitzpatrick, J. A., 1988, “A Generalized Technique for Spectral Analysis of Nonlinear Systems," Mechanical Systems and Signal Processing, Vol. 2, pp. 195-207.

Rice, H. J., and Fitzpatrick, J. A., 1991, “A Procedure for the Identification of Linear and Nonlinear Multi-Degree-Of-Freedom Systems," Journal of Sound and Vibration, Vol. 149, pp. 397-411.

Rugh, W. J., 1981, Nonlinear System Theory: The Volterra/Wiener Approach, Johns Hopkins Press, Baltimore, MD.

Schetzen, M., 1980, The Volterra \& Wiener Theories of Nonlinear Systems, Wiley-Interscience, NY.

Vugts, J. S., and Bouquet, A. G., 1985, “A Nonlinear Frequency Domain Description of Wave Forces on an Element of a Vertical Pile,' Proceedings, Behavior of Offshore Structures, pp. 239-259. 

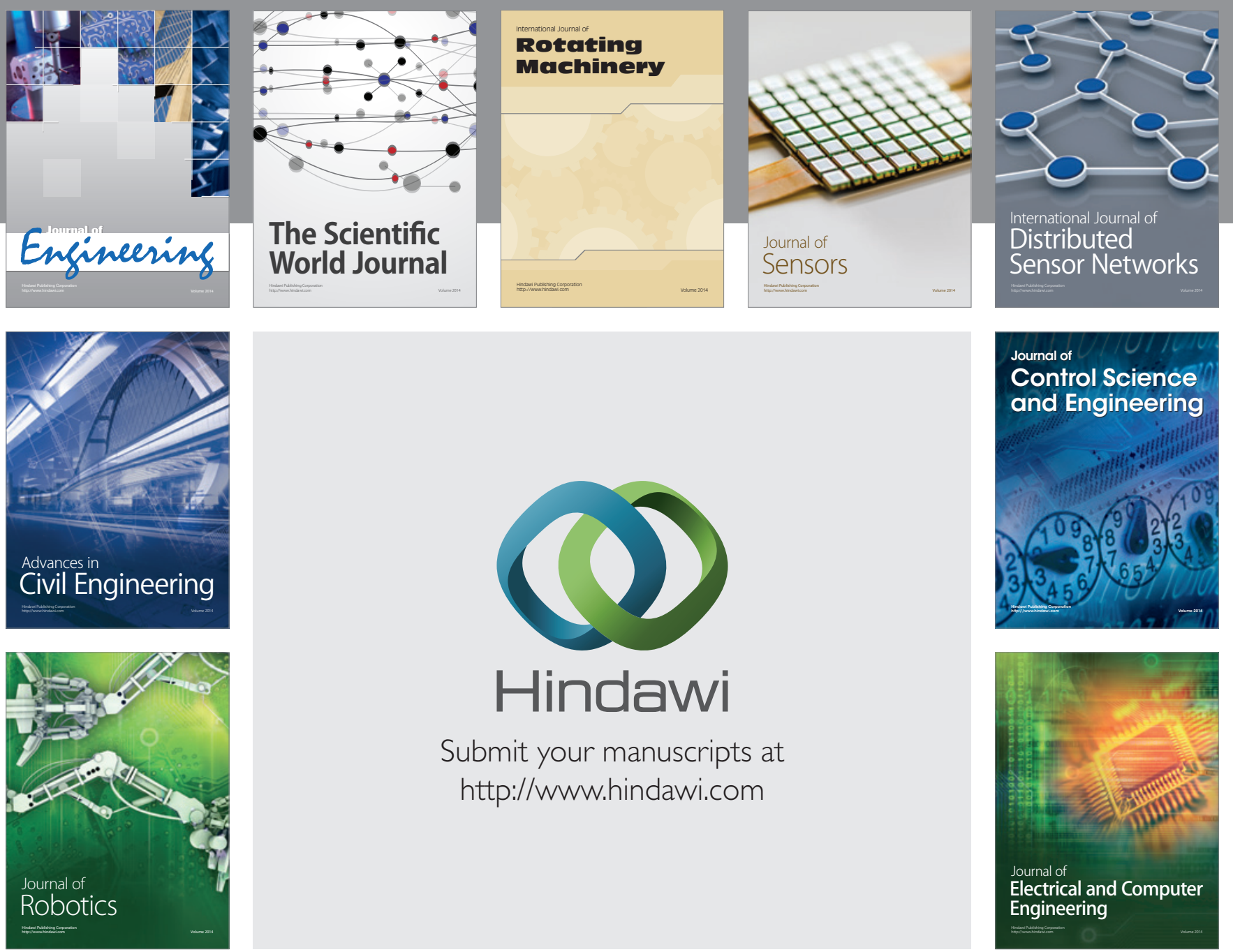

Submit your manuscripts at

http://www.hindawi.com
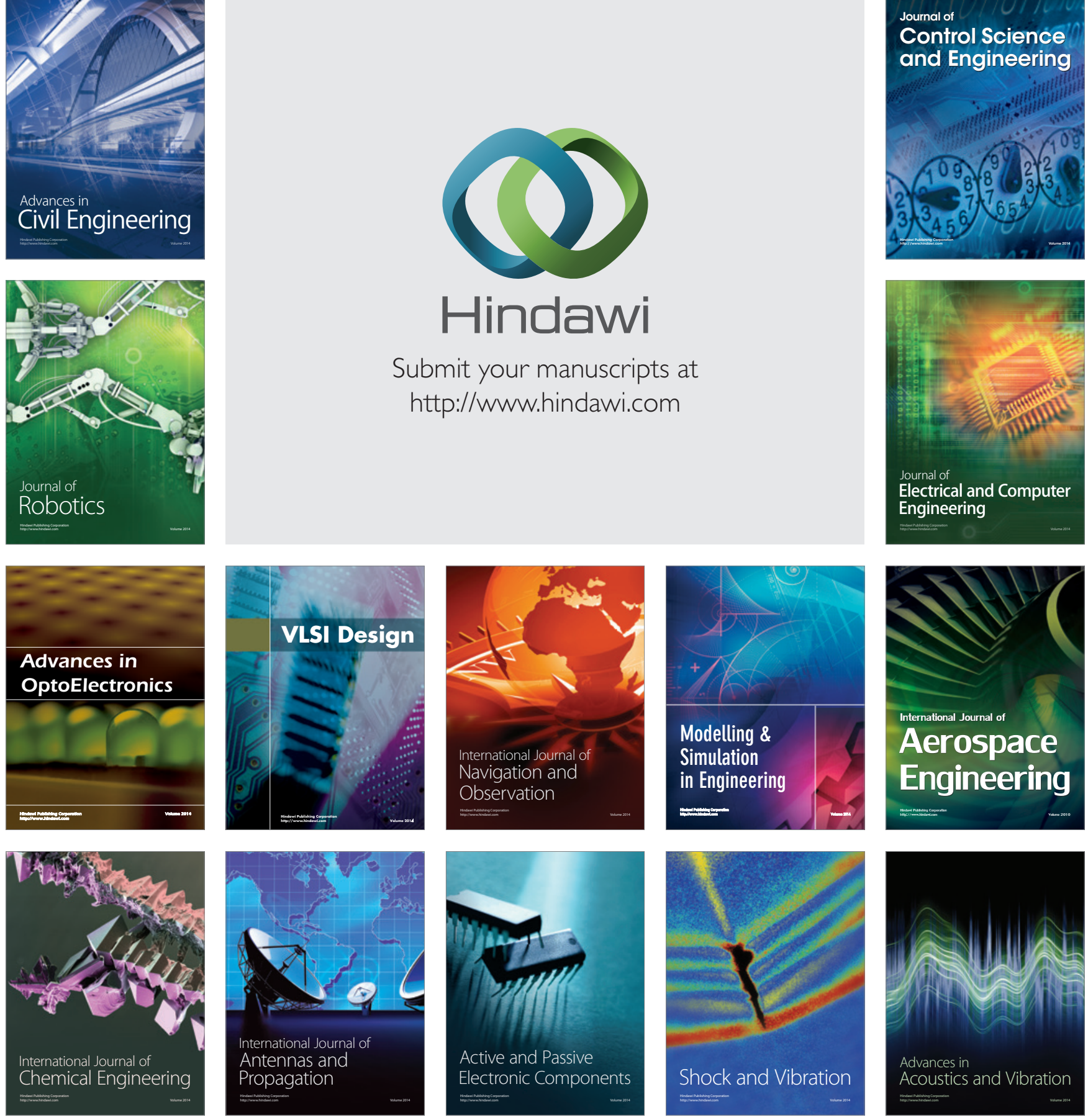